\title{
Prevalence, pattern and perceptions of self-medication in medical students
}

\author{
Rushi N. Pandya ${ }^{1}$, Kunal S. Jhaveri ${ }^{2}$, Falgun I. Vyas ${ }^{3}$, Varsha J. Patel $^{4} *$
}

${ }^{1}$ Resident, Department of Pharmacology, Smt. NHL Muni. Medical College, Ahmedabad, Gujarat, India

${ }^{2}$ Medical Advisor, AstraZeneca, Bengaluru, India

${ }^{3}$ Medical Advisor, Intas

Pharmaceutical, Ahmedabad, India

${ }^{4}$ Professor \& Head, Department of Pharmacology, Smt. NHL Muni. Medical College, Ahmedabad, Gujarat, India

Received: 23 April 2013

Accepted: 5 May 2013

*Correspondence to:

Dr. Varsha J. Patel,

Email: drvarsha4@rediffmail.com

(C) 2013 Pandya RN et al. This is an open-access article distributed under the terms of the Creative Commons Attribution License, which permits unrestricted use, distribution, and reproduction in any medium, provided the original work is properly cited.

\begin{abstract}
Background: The use of self-medication is highly prevalent in the community more so amongst the medical students. Self-medication can be defined as the use of drugs to treat self-diagnosed disorders or symptoms, or the intermittent or continued use of a prescribed drug for chronic or recurrent disease or symptoms.

Aims and Objectives: To study the prevalence and pattern of use of selfmedication among medical students from first year to internship.

Methods: This cross sectional study was carried out among under graduate medical students including interns of Smt. NHL Municipal Medical College, Ahmedabad during the period of March 2010 to May 2010.

Results: Out of 747 students and interns enrolled, 685 responded (91.7\%). Out of 685 respondents $564(82.3 \%)$ reported self-medication within one year of recall period. Most common conditions/symptoms for selfmedication in students were fever $(72.7 \%)$, headache $(69.1 \%)$, upper respiratory tract infections $(64.1 \%)$ followed by others like body-ache, abdominal pain, diarrhoea etc. Over the counter drugs $(84.2 \%)$ was the most common category of drugs used by all the students except first year students who used prescription only drugs more frequently $(48.5 \%)$. Herbal and Ayurvedic drugs were also used as self-medication (17.8\%); most frequently by the first year students $(22.7 \%)$.

Conclusion: The pattern of self-medication practice changes with time and advancement of knowledge.
\end{abstract}

Keywords: Self-medication, Medical students, OTC drugs, Survey

\section{INTRODUCTION}

Self-medication can be defined as the use of drugs to treat self-diagnosed disorders or symptoms, or the intermittent or continued use of a prescribed drug for chronic or recurrent disease or symptoms. In practice, it also includes use of the medication of family members, especially where the treatment of children or the elderly is involved. ${ }^{1}$ Use of self-medication is highly prevalent in both urban and rural community varying from $32.5 \%$ to $81.5 \% .^{2-4}$ Self-prescribing among doctors is reported to be prevalent. In a study of self medication in young Norwegian doctors, $56 \%$ of hospital physicians and the general practitioners reported self-medication during the previous year. ${ }^{5} \mathrm{~A}$ quarter of the primary healthcare professionals in Brazil self-medicated themselves. ${ }^{6}$ Self-medication with antibiotics was reported by $53 \%$ of doctors in a study from Karnataka state of India. ${ }^{7}$
The practice of self-medication among doctors develops during their training period as obvious from some studies of self-medication among medical students. These studies have reported high prevalence ranging from $57.7 \%$ to $76 \%{ }^{8,9}$ Self-medication is also reported to be quite popular among Indian medical students. ${ }^{10}$ However there is a paucity of studies of the prevalence and pattern of self medication among medical students from India. This questionnaire based study was planned to find out the prevalence and pattern of self-medication in undergraduate medical students and their perceptions about self-medication.

\section{METHODS}

This cross sectional study was carried out among under graduate medical students including interns of Smt. NHL Municipal Medical College, Ahmedabad during the period of March 2010 to May 2010. The study began 
after approval of the Institutional Ethics Committee (IEC).

Data were collected through structured, validated 12 questions based questionnaire which was filled up by students after explaining about the aim of study and giving assurance about confidentiality of all information. Data were analysed for those students who had self medicated within one year of recall period.

The questionnaire included questions pertaining to demographic details, professional year of the M.B.B.S. course (first year, second year, third year part 1, third year part 2 or internship), frequency of self medication during one year recall period, indications for selfmedication, details of type and pattern of drug usage, sources of drug information used, use of psychotropic agents as self medication, reasons for favoring selfmedication and knowledge of adverse effects of drugs used.

Data were analysed using MS Excel 2007.

\section{RESULTS}

Out of 747 students and interns enrolled, 685 responded $(91.7 \%)$. Out of 685 respondents $564(82.3 \%)$ reported self medication within one year of recall period. Table 1 shows the socio-demographic characteristics of the 685 students who responded irrespective of self-medication within one year recall period.

Out of 564, $371(65.9 \%)$ had self-medicated with frequency of 1-5 times in last year and self-medication was highest in the second year students with 89 (74.8\%), while lowest in interns with $47(41.6 \%)$. Fifty seven $(10.1 \%)$ students had self medicated more than 12 times in one year recall period.

Most common conditions/symptoms for self-medication in students were fever (72.7\%), headache (69.1\%), upper respiratory tract infections $(64.1 \%)$ followed by others like bodyache, abdominal pain, diarrhoea etc (Figure 1). For majority of symptoms intern doctors resorted to self medication, while lowest use was reported by first year medical students.

Over the counter drugs (84.2\%) was the most common category of drugs used by all the students except first year students who used prescription only drugs more frequently $(48.5 \%)$. Herbal and Ayurvedic drugs were also used as self medication (17.8\%); most frequently by the first year students (22.7\%) (Figure 2). The most commonly used drugs for self-medication were paracetamol $(65.6 \%)$, anti infective agents $(44.7 \%)$, NSAIDS $(34.7 \%)$ followed by $\mathrm{H} 1$ antihistaminics (25.8\%), gastrointestinal drugs $(13.1 \%)$ and herbal and Ayurvedic drugs (7.8\%). Third year MBBS students were the most frequent users of self medication for majority of drugs including anti-infectives which are POMs except interns for psychotropic drugs and first year students for herbal and Ayurvedic drugs (Figure 3).

The important sources of information for self-medication were relatives and physicians (48.6\%), seniors and friends $(45.4 \%)$ and textbook and related materials $(44.5 \%)$. The frequency of use of textbooks and related printed material as major source of information increased from first year $(6.2 \%)$ to third year students $(76.3 \%)$.

Table 1: Demographic profile of medical students $(n=685)$.

\begin{tabular}{|lllll|l|}
\hline $\begin{array}{l}\text { Professional } \\
\text { Year -MBBS }\end{array}$ & $\begin{array}{l}\text { Respondents } \\
\text { No. }\end{array}$ & $\begin{array}{l}\text { Self Medicated in } \\
\text { Last Year } \\
\text { No. }(\%)\end{array}$ & $\begin{array}{l}\text { Male } \\
\text { No. }(\%)\end{array}$ & $\begin{array}{l}\text { Female } \\
\text { No. }(\%)\end{array}$ & $\begin{array}{l}\text { Age -years } \\
\text { Mean } \pm \text { S.E. }\end{array}$ \\
\hline First & 139 & $97(69.80)$ & $89(64.00)$ & $50(36.00)$ & $17.88 \pm 0.631$ \\
\hline Second & 160 & $119(74.37)$ & $94(58.75)$ & $66(41.25)$ & $19.28 \pm 0.632$ \\
\hline Third- Part 1 & 136 & $117(86.00)$ & $92(67.60)$ & $44(32.40)$ & $19.74 \pm 0.599$ \\
\hline Third- Part 2 & 131 & $118(90.10)$ & $97(74.00)$ & $34(26.00)$ & $21.09 \pm 0.561$ \\
\hline Interns & 119 & $113(95.00)$ & $96(80.70)$ & $23(19.30)$ & $22.92 \pm 0.570$ \\
\hline Total & 685 & $564(82.30)$ & $468(68.30)$ & $217(31.70)$ & $20.02 \pm 0.069$ \\
\hline
\end{tabular}




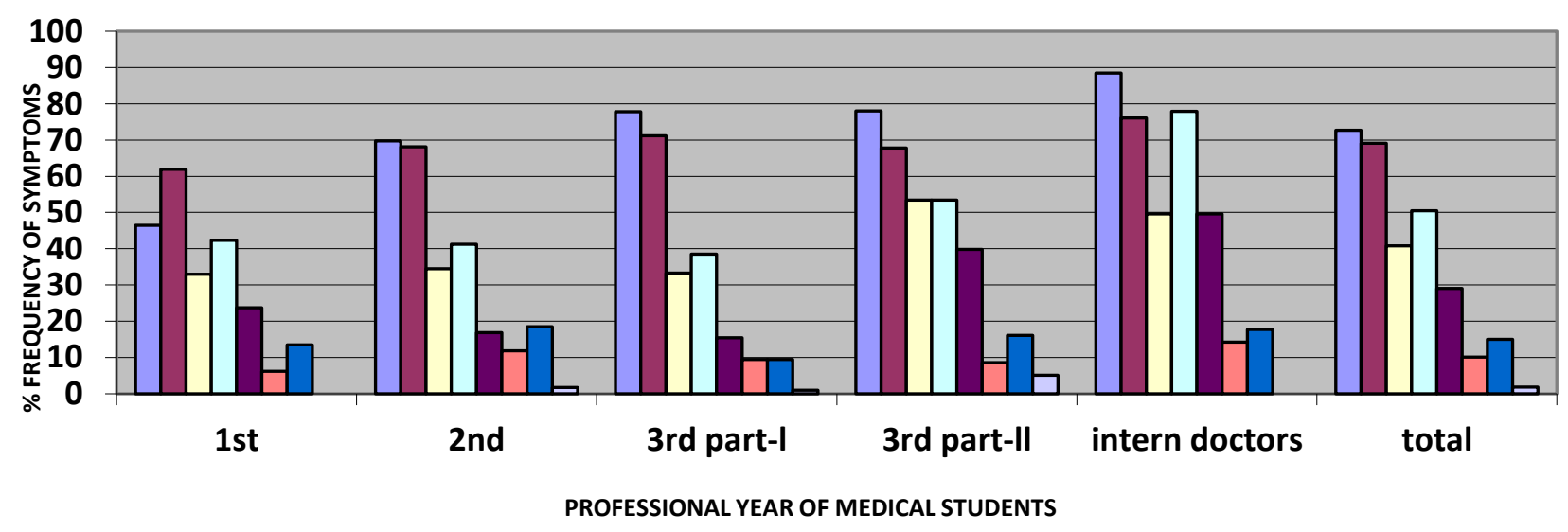

\begin{tabular}{|llll|}
\hline$\square$ Fever & $\square$ Headache & $\square$ Body ache & $\square$ Cough \\
$\square$ Diarrhoea & $\square$ Constipation & $\square$ Abdominal Pain & $\square$ Other symptoms \\
\hline
\end{tabular}

Figure 1: Conditions/symptoms for self medication.

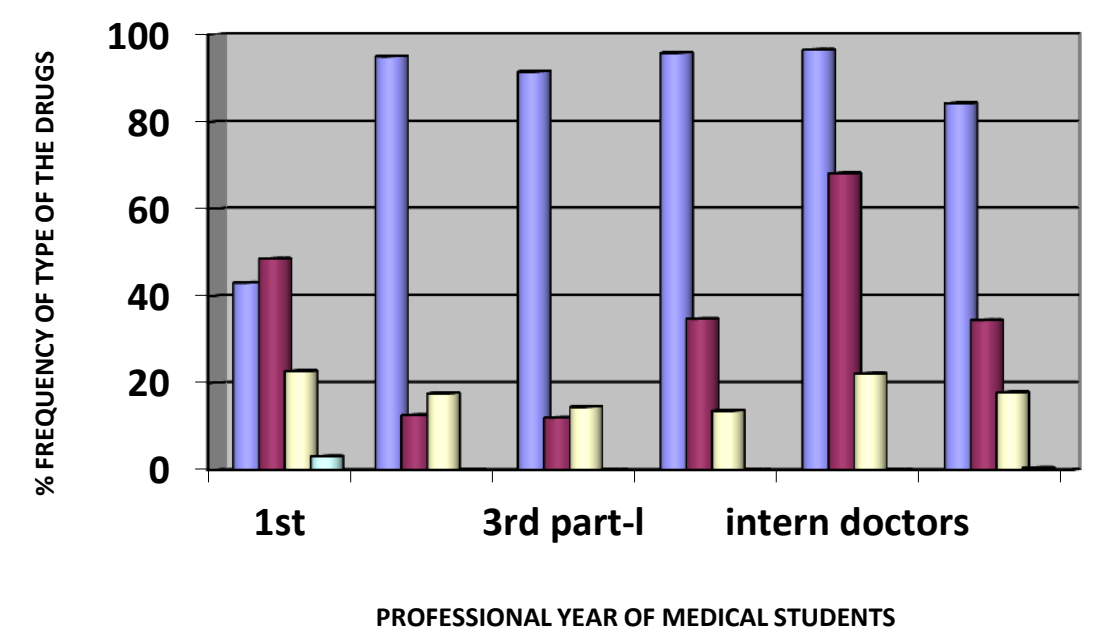

口O.T.C. medicines* $\square$ P.O. medicines** $\square$ herbal / ayurvedic medicines $\square$ others

Figure 2: Drug categories used for self medication by medical students.

(* Over the counter medicines, ** Prescription only medicines)

Total $95(16.8 \%)$ students used psychotropic drugs as self-medication, out of which $13.9 \%$ of students were using sedatives-hypnotics and antidepressants. Use of sedatives-hypnotics and antidepressants increased with seniority from $4.1 \%$ in first year to $24.5 \%$ among interns. There was a significant association of self-medication with Out of 564 responders, $409(72.3 \%)$ felt that self- medication frequency had increased progressively with education level. About 70\% (398) students opined in favor of self-medication. Most common reasons for favoring self-medication were time saving (41.2\%) followed by convenience $(39.6 \%)$, quick relief $(35.5 \%)$ and cost-effectiveness (22.8\%) (Table 2). 


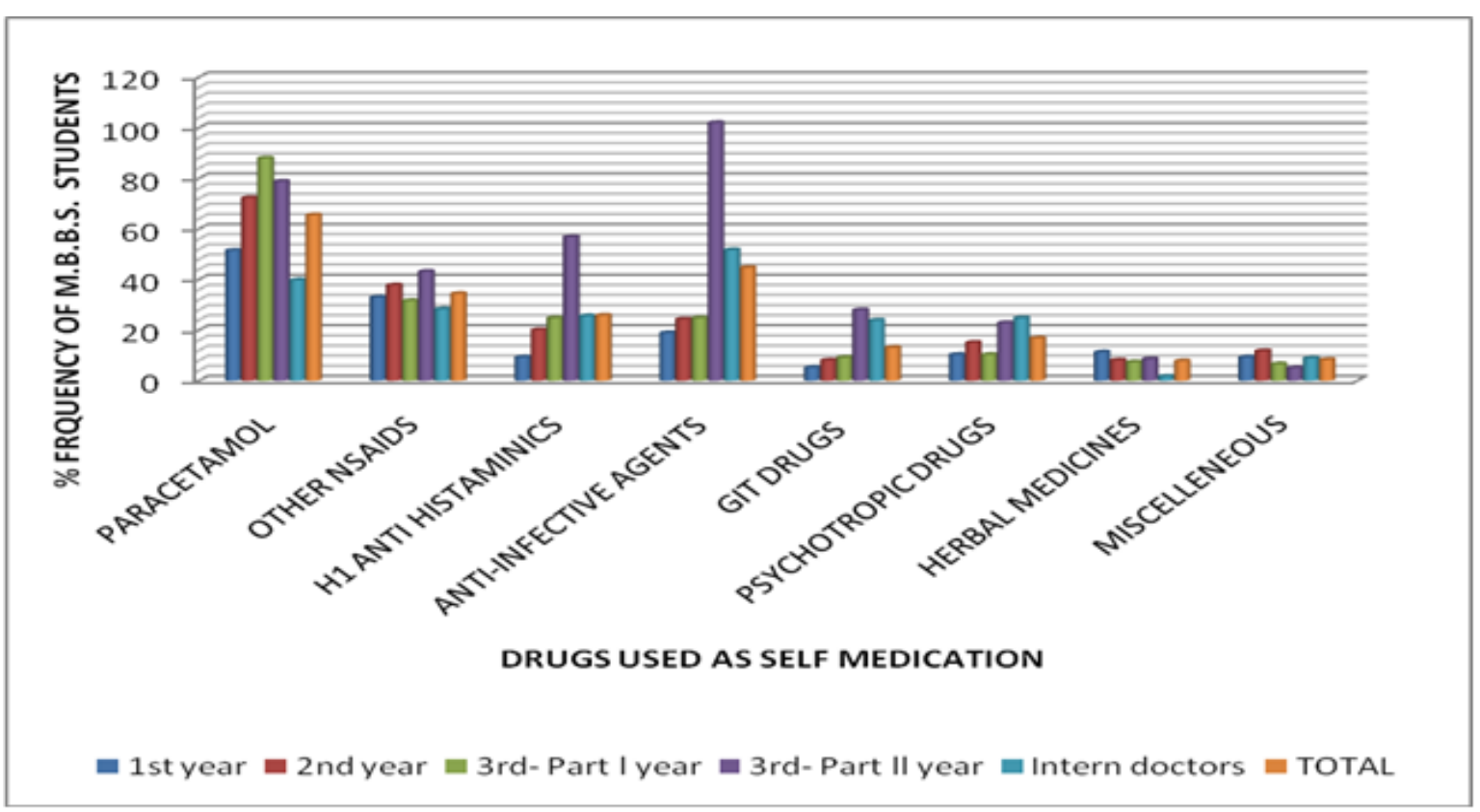

Figure 3: Drugs used for self-medication by medical students.

Table 2: Reasons for favoring self medication.

\begin{tabular}{|ll|}
\hline Reason & No. $(\%)$ of respondents \\
\hline Time saving & $233(41.20)$ \\
\hline Convenience & $224(39.60)$ \\
\hline Quick relief & $201(35.50)$ \\
\hline Economical & $129(22.80)$ \\
\hline Others & $06(01.10)$ \\
\hline
\end{tabular}

Out of 564 respondents, 401 (70.8\%) students were aware of adverse reactions of drugs used for self medication, while $43(7.6 \%)$ students had experienced it.

There was significant association of self-medication with increased level of education $(p=0.001)$ and examination stress. $(p=0.003)$. There was no significant association of self-medication with gender.

\section{DISCUSSION}

The practice of self-medication is widespread all over the world especially urban and educated population., ${ }^{2,3}$ Doctors also tend to self medicate to the extent feasible due to their knowledge about both diseases and drugs. Several studies have reported prevalence of selfmedication varying from 25-56\%., Medical students acquire knowledge of drugs and skills of drug selection and prescribing during the undergraduate training which includes internship in many countries including India. It is also known that this group starts relying on self medication during their formative years of undergraduate training. Some studies have reported prevalence and pattern of self-medication in medical students with varying results. ${ }^{10,11}$

Our results suggest a high prevalence $(82 \%)$ of selfmedication in our students which is somewhat higher than reported by Zafar et al who studied self medication in medical and non medical students and found no significant difference between the two groups ${ }^{9}$ and lower than reported by Badiger et al reporting $92 \%$ prevalence in a study of 200 students. ${ }^{12}$ The most important reason for higher trend of self medication is easy availability of all categories of medicines- OTC, prescriptions only or even schedule $\mathrm{X}$ drugs (Narcotics) without prescription to consumers in India. As a result high prevalence has been reported even in urban population. $^{2,3}$ The situation is much more favorable for medical students. Other factors favoring self medication are acquired knowledge of medicines, convenience and saving time due to no need of consulting a doctor.

In spite of high prevalence, the frequency of self medication is not high with about $70 \%$ reporting 1 to 5 times in one year recall period. Moreover, over the counter drugs is the most frequently used category meaning they exercise discretion when using POM as self medication. It is also observed in this study that most of self medication is for minor common ailments like fever, headache, cough etc which is in accordance with previous studies in University students. ${ }^{9,12}$ Use of drugs from 
alternative medicine (herbal, Ayurvedic) was also reported, more frequently by first professional year students. This could be due to continuation of self medication practices in urban population in general in their first year of medical education. This trend decreases with their increasing knowledge and self confidence in modern medicines. A study from China has also reported frequent use of Chinese herbal medicines by University students. $^{13}$

More than $90 \%$ of respondents were aware of the adverse affects associated with self-medication as compared to just above $30 \%$ in the study by Badiger et al. ${ }^{12}$

There was an increasing trend of use of anti-infectives from first year to third year students. A recent study from India also found significantly higher number of third students using drugs for treatment of infections than first year students. ${ }^{14}$

Our study found that while important sources of information regarding drugs are relatives and physicians, seniors and friends and textbook and related materials. However the frequency of use of textbooks and related materials major source of information increased from first year $(6.2 \%)$ to third year students $(76.3 \%)$ suggesting that medical students rely more and more on the objective sources of information with progressive gain in knowledge about medicines.

Self-medication with psychotropic drugs is reported by about $17 \%$ of respondents. Still the prevalence is not small and also expected due to high degree of stress in life of medical students. Stress was found to be significantly more in Second and Third MBBS students than First MBBS levels. ${ }^{15}$ Our study also found increasing use of psychotropic drugs in second and third year students except third year part 1 which reported less frequent use than second year students. There could be under reporting by the students as some may not want to reveal this sensitive information in spite of responses being obtained anonymously.

In a review of self medication in physicians and medical students the authors concluded that the concept of treating one's own illnesses without going through the usual channels patients normally follow (doctor selftreatment) is deeply ingrained in medical culture and is acquired as early as when aspiring physicians are medical students. ${ }^{16}$ Previous studies have included both medical $^{7,10}$ and nonmedical students ${ }^{9}$, carried out survey in first year students, first and second year students or first and third year students. Our study has included majority of students from first year to interns. Hence this study can better reflect the practices and change in the pattern with advancement of students in their studies.

The strength of the study is high response rate with 564 out of total 685 students from first professional year to internship participating in the study.

\section{CONCLUSION}

To conclude the practice of self medication is highly prevalent in medical students with majority restricting the use to treatment of minor ailments with over the counter drugs. The pattern of self medication practice changes with time and advancement of knowledge.

Funding: No funding sources

Competing interests: None declared

Ethical approval: The study was approved by the Institutional Ethics Committee (IEC)

\section{REFERENCES}

1. World Health Organization: Guidelines for the regulatory assessment of Medicinal Products for use in self-medication. WHO/EDM/QSM/00.1, 2000.

2. Lam CL, Catarivas MG, Munro C, Lauder IJ. Selfmedication among Hong Kong Chinese. Soc Sci Med 1994;39:1641-7.

3. Sanghani S, Zaveri HG, Patel V J. Self medication: Prevalence and Pattern in urban community. J Pharmacovigilance Drug Safety 2008;5:95-8.

4. Phalke VD, Phalke DB, Durgawale PM. SelfMedication Practices in Rural Maharashtra. Indian J Community Medicine 2006;31:34-5.

5. Hem E, Stokke G, Tyssen R, Grønvold N T, Vaglum $\mathrm{P}$, Ekeberg O. Self-prescribing among young Norwegian doctors: a nine-year follow-up study of a nationwide sample. BMC Med 2005;3:16.

6. Graciela ET, Castro SA, Oppelt AM, Petrini RM, Pereira IV, Sassi BT. Working conditions and selfmedication among primary healthcare professionals in an urban area of Pelotas, RS. Rev bras Epidemiol 2007;10:66-74.

7. Nalini GK. Self-Medication among Allopathic medical Doctors in Karnataka, India. BJMP 2010;3:325.

8. James H, Handu SS, Khaja KA, Sequeira RP. Influence of medical training on self-medication by students. Int J Clin Pharmacol Ther 2008;46;23-9.

9. Zafar SN, Syed R, Waqar S, Zubairi AJ, Vaqar T, Shaikh M, Yousaf W, Shahid S, Saleem S. Selfmedication amongst University Students of Karachi: Prevalence, Knowledge and Attitudes. J Pak Med Assoc 2008;58:214-8.

10. Gupta YK. Popularity of self medication among medical students, AIIMS. Available at www.INDIAedunews.net. Accessed 19 January 2013.

11. Abay SM, Amelo W. Assessment of SelfMedication Practices Among Medical, Pharmacy, and Health Science Students in Gondar University, Ethiopia. J Young Pharm 2010;2:306-10.

12. Badiger S, Kundapur R, Jain A, Kumar A, Pattanshetty S, Thakolkaran N, Bhat N, Ullal N. Self-medication patterns among medical students in South India. Australas Med J 2012;5:217-20. 
13. Lau GS, Lee KK, Luk CT. Self-medication among university students in Hong Kong. Asia Pac J Public Health 1995;8:153-7.

14. Sontakke SD, Bajait CS, Pimpalkhute SA, Jaiswal KM, Jaiswal SR. Comparative study of evaluation of self-medication practices in first and third year medical students. Int J Biol Med Res 2011;2:561-4.
15. Supe AN. A study of stress in medical students at Seth G.S. Medical College. J Postgrad Med 1998;44:1-6.

16. Montgomery AJ, Bradley C, Rochfort A, Panagopoulou E. A review of self-medication in physicians and medical students. Occup Med (Lond) 2011;61:490-7.

doi:10.5455/2319-2003.ijbcp20130608

Cite this article as: Pandya RN, Jhaveri KS, Vyas FI, Patel VJ. Prevalence, pattern and perceptions of selfmedication in medical students. Int J Basic Clin Pharmacol 2013;2:275-80. 